\title{
Essential Anatomy of the Anorectum for Colorectal Surgeons Focused on the Gross Anatomy and Histologic Findings
}

\author{
Jong Min Lee, Nam Kyu Kim \\ Division of Colorectal Surgery, Department of Surgery, Colorectal Cancer Clinic, Severance Hospital, Yonsei University College of Medicine, \\ Seoul, Korea
}

The anorectum is a region with a very complex structure, and surgery for benign or malignant disease of the anorectum is impossible without accurate anatomical knowledge. The conjoined longitudinal muscle consists of smooth muscle from the longitudinal muscle of the rectum and the striate muscle from the levator ani and helps maintain continence; the rectourethralis muscle is connected directly to the conjoined longitudinal muscle at the top of the external anal sphincter. Preserving the rectourethralis muscle without damage to the carvernous nerve or veins passing through it when the abdominoperineal resection is implemented is important. The mesorectal fascia is a multi-layered membrane that surrounds the mesorectum. Because the autonomic nerves also pass between the mesorectal fascia and the parietal fascia, a sharp pelvic dissection must be made along the anatomic fascial plane. With the development of pelvic structure anatomy, we can understand better how we can remove the tumor and the surrounding metastatic lymph nodes without damaging the neural structure. However, because the anorectal anatomy is not yet fully understood, we hope that additional studies of anatomy will enable anorectal surgery to be performed based on complete anatomical knowledge.

Keywords: Anorectum anatomy; Pelvic fascia; Pelvic plexus; Neurovascular bundles

\section{INTRODUCTION}

Surgical procedures for both benign and malignant disease of the anorectum are known to be technically demanding procedures. As far as the field of anorectal surgery is concerned, not only the short-term outcome but also the long-term functional outcome is a very important issue, probably due to the complexity of the anorectal anatomy. Both minor and major surgical procedures have real impacts on the quality of life of patients.

Because surgical techniques are based on an understanding of anatomy, accurate anatomical knowledge of the anorectum helps

Received: November 14, 2017 - Accepted: December 15, 2017

Correspondence to: Nam Kyu Kim, M.D.

Division of Colorectal Surgery, Department of Surgery, Colorectal Cancer Clinic, Severance Hospital, Yonsei University College of Medicine, 50-1

Yonsei-ro, Seodaemun-gu, Seoul 03722, Korea

Tel: +82-2-2228-2100, Fax: +82-2-313-8189

E-mail: NAMKYUK@yuhs.ac

ORCID code: https://orcid.org/0000-0003-0639-5632

(C) 2018 The Korean Society of Coloproctology

This is an open-access article distributed under the terms of the Creative Commons Attribution NonCommercial License (http://creativecommons.org/licenses/by-nc/4.0) which permits unrestricted noncommercial use, distribution, and reproduction in any medium, provided the original work is properly cited. the surgeon to perform safe, high-quality surgery on patients with rectal diseases ranging from benign to malignant. Especially in rectal cancer surgery, an approach to the anus, such as the transanal total mesorectal excision, is attempted at a location different from that used in the conventional transabdominal approach, so a full understanding of the anatomy around the anus is now needed. Also, familiarity with the techniques used to treat patients with benign anorectal diseases, such as the hemorrhoidectomy or the fistulectomy, will be useful to surgeons performing coloanal or intersphincteric procedures for the surgical treatment of patients with distal rectal cancer. For those reason, we will discuss in this paper the basic anatomy of the anorectum and the latest knowledge on that subject. We will also describe the important structures and surgical strategies from the surgeon's point of view.

\section{OVERALL ANATOMY OF THE ANAL CANAL}

The anatomic anal canal is defined as beginning at the dentate line and ending at the anal verge. On the other hand, the surgical anal canal is defined as the area between the anorectal ring and the anal verge. The anorectal ring is usually palpable as a tight ring-like structure, and when patients are asked to squeeze the 
anal sphincter, it moves anteriorly. The anorectal ring consists of the external anal sphincter and the puborectalis muscles and is a good landmark for locating the tumor or the level of the colorectal anastomosis. The length of the surgical anal canal is $4.4 \mathrm{~cm}$ in men compared with $4.0 \mathrm{~cm}$ in women [1]. The internal anal sphincter (IAS), external anal sphincter and puborectalis muscle constitute the surgical anal canal. The anorectal ring is easily identified on digital examination and on image studies such as transrectal ultrasound or rectal magnetic resonance imaging (MRI).

The anal canal has a cylindrical double-layered shape. The inner layer is composed of the IAS and the conjoined longitudinal muscle (CLM), which is innervated by the autonomic nerve system, whereas the outer layer is composed of the puborectalis muscle and the external sphincter muscle (EAS), which is stimulated by somatic nerves. The EAS finishes more distally than the internal sphincter. The EAS constitutes the end of the anal canal, just as it surrounds the IAS from the outside to the inside. As the orientation of the anal canal changes, the shape of the IAS, EAS, and CLM that compose anal canal also change. According to one study of the anal canal, which divided the anal canal into 9 different sagittal planes, the levator ani muscle in the anterolateral portion of anal canal is relatively thick and straight while the EAS is thin. In the lateral portion, the levator ani muscle is thick and curved whereas in the posterior portion, the levator ani muscle is thin and curved, and the EAS is thick. In contrast, the attachment length from the levator ani muscle to the CLM decreases as opposed to the gradual increase in the fraction of the levator ani muscle that overlaps the EAS from the anterolateral to the posterior position [2]. The anal canal ends at the anal verge, forming a transitional zone between the epithelium and the perianal skin. From a practical point of view, the grove between the internal and the external anal sphincter can be easily palpitated under anesthesia and should serve as a landmark for an internal sphincterotomy for anal fissure or an intersphincteric resection (ISR) for low-lying rectal cancer.

\section{ANAL CANAL'S EPITHELIUM}

The dentate line is the most important landmark for understanding the epithelium of the anal canal. The dentate line is located one third below the entire surgical anal canal. The area between the dentate line and the anal verge is called the "anoderm," which is a nonkeratinized squamous epithelium and has no structures such as hair follicles or glands. It is also soft when palpated and sensitive to pain. During a hemorrhoidectomy, the excised portion is usually excessive or increased anoderm. When the anoderm is resected excessively, anal stricture may occur. Avoiding the underlying internal and external anal sphincter during excision of the anoderm and vascular cushions is also important. About $10 \mathrm{~mm}$ proximal to the dentate line, the "anal transitional zone" is found. It appears purple and represents an area of gradual transition from a squamous epithelium to a columnar epithelium.
The transitional zone is known to be associated with the sampling reflex, which means that this area can discriminate feces from gas. Nevertheless, its functional role is still not clear.

The column of Morgagni begins at the dentate line, and the anal crypts are at its base. The anal crypts are connected to the underlying anal glands through the anal gland duct, and these are the presumed source of perianal abscesses and perianal fistulae. The anal gland ducts proceed from the anal canal and enter the submucosa, where two-thirds enter the IAS and half terminate in the intersphincteric plane [3]. The obstruction of these ducts is postulated to lead to anal fistulae and abscesses, which is the reason the internal opening of the cryptoglandular anal fistula is located at the dentate line. When a surgeon plans to perform a total proctectomy and coloanal anastomosis (CAA), the dividing line will be at the anorectal junction or at the dentate line (stapled CAA or hand-sewn CAA). The surgeon should consider the glandular ducts around there. With our initial CAA procedure, we experienced a couple of unexplained perianal fistulae.

Distal to the dentate line, the anoderm begins and continues for about $1.5 \mathrm{~cm}$. At the anal verge, the squamous cells merge with the perianal skin. The perianal skin is also made up of squamous cells, but it has hair follicles and glands. The area below the dentate line is innervated with the inferior rectal nerve, which makes us sensitive to pain. However, the region proximal to the dentate line is innervated with the autonomic nerve system. This difference can be explained by the dentate line being a margin of different embryonic origins (endoderm vs. ectoderm).

\section{INTERNAL ANAL SPHINCTER}

When the rectum inserts into the pelvic diaphragm, the inner circular muscle of the rectum continues to the IAS and terminates approximately $1 \mathrm{~cm}$ proximal to the distal edge of the EAS. Overall, the IAS is found to be approximately $2-3 \mathrm{~mm}$ in thickness [4] and to become thicker near the anal verge. Both the sympathetic fibers from the inferior pelvic plexus and the parasympathetic fiber through the inferior pelvic plexus and splanchnic nerves (S24) innervate this muscle [5]. An anorectal manometric study showed that constant resting pressure may reflect the tone of the IAS. With solid stool in the rectum, the IAS begins to relax, and the external anal sphincter begins to contract; this is called the rectoanal inhibitory reflex.

\section{CONJOINED LONGITUDINAL MUSCLE}

The CLM, also called the longitudinal anal muscle, has been described as a vertical layer of muscular tissue interposed between the IAS and the EAS. This muscle lies on the intersphincteric space, which contains a thin layer of fatty tissue. The CLM begins at the anorectal ring as an extension of the longitudinal rectal muscle fibers and descends caudally [6]. Measuring approximately $2.5 \mathrm{~mm}$ in thickness, the CLM decreases in thickness with 
Volume 34, Number 2, 2018

age [4], but there is no general agreement on its composition and attachments.

According to Macchi et al. [7], the CLM is mainly composed of outer striated muscle fibers and small numbers of inner smooth muscle fibers, which, based on immunohistochemical staining studies, originate from the levator ani muscle and the longitudinal muscle of the rectum. They also noted the presence of an oblique muscle bundle that went from the EAS to the IAS and vice versa. The spatial outcome was the spiral course of the striated and the smooth muscle fibers between the EAS and the IAS, which contributed to some shortening of the anal canal as well as to a narrowing of the anal canal during sphincter contraction (Fig. 1). Now, we can speculate that this muscle may contribute to maintaining continence. Moreover, Shafik [8] hypothesized that this muscle played a role in defecation. When the pubococcygeus contracts, the CLM also contracts, which makes the anal canal shorter and wider.
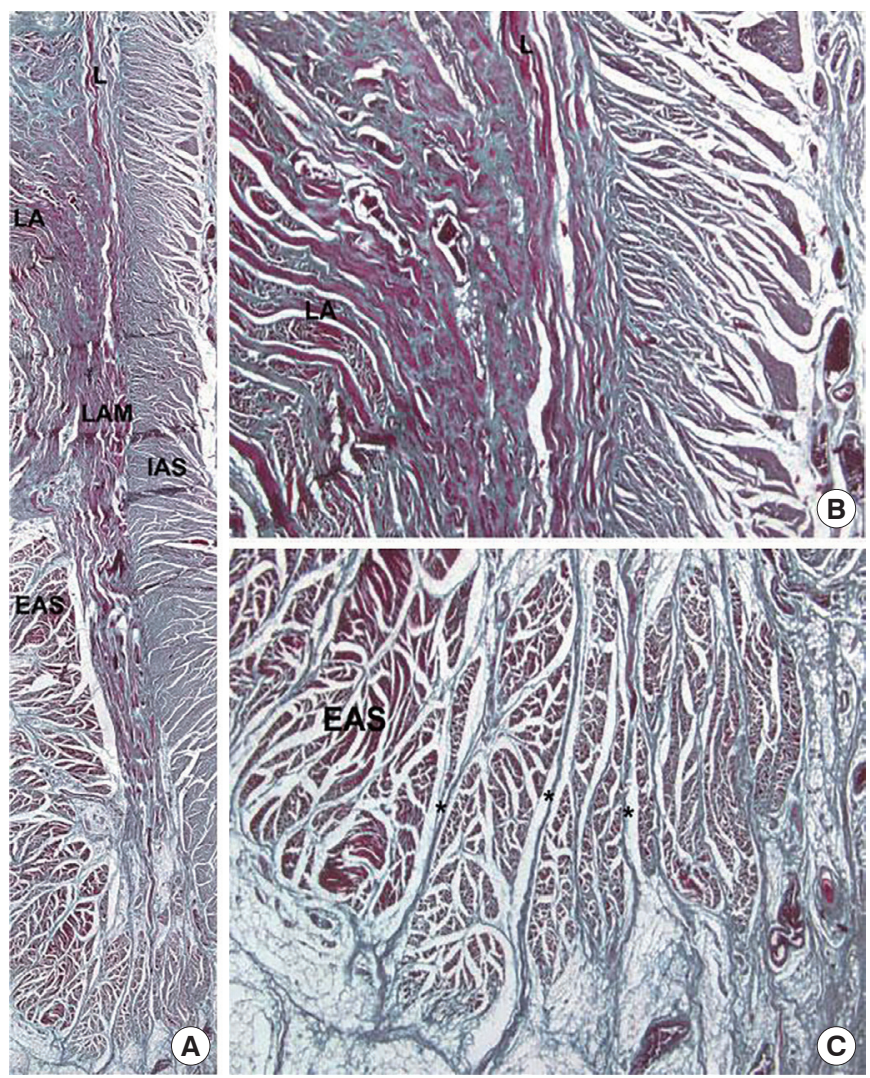

Fig. 1. (A) The coronal section of the anal canal shows the LAM between the IAS and the EAS. (B) The continuation of the LA to the LAM is shown. (C) The LAM terminates with fibroelastic septa (asterisks) (azan Mallory staining, $\times 2.5$ ); L, longitudinal muscle of the rectum; LA, levator ani; LAM, longitudinal anal muscle; EAS, external anal sphincter; IAS, internal anal sphincter. Reproduced from Macchi et al. Clin Anat 2008;21:447-52, with permission of John Wiley and Sons [7].
The anatomy of the CLM in relation to the IAS and the EAS is very important in implementing an ISR for low rectal cancer. The distal resection line may be at the intersphincteric groove (total ISR), or between the dentate line and the intersphincteric groove (subtotal ISR), or at the dentate line (partial ISR) [9]. According to Valadão et al. [10], a resection of the proximal half of the internal sphincter should be considered when the incision is made on the dentate line or 1 to $2 \mathrm{~mm}$ distal to it. If the resection is initiated above the dentate line, but below the anorectal junction, a proximal third removal of the internal sphincter should be considered. Regardless of where the surgeon enters, the internal sphincter should be resected following the intersphincteric plane including the CLM. At the top of the EAS is the rectourethral muscle (RUM), which is on the link between the longitudinal muscle of the rectum coming from below and Denonvillers' fascia (DVF) coming from above. Because the RUM is known to be the tissue through which a vein and the cavernous nerve pass, complications can be prevented by dissecting into the back of this muscle [11]. In the lateral part of the ISR, the perineal plane tends to be directed dorsally to the levator ani muscle due to the overlap of levator ani muscle and the EAS. This causes a mismatch between the abdominal plane and the perineal plane. However, in this case, if the surgeon just cut the levator ani muscle between the 2 planes and that lead surgeon to complete the ISR plane [2]. The same concept can be applied for a hemilevator excision followed by an ISR for treating a tumor involving the puborectalis muscle [12]. When an ISR is planned, the surgeon should realize that the CLM may be completely broken, which can affect the patient's continence. Köhler et al. [13] reported a 29\% reduction in resting anal pressure following an ISR; the squeeze pressure recovered to preoperative levels after 12 months.

At the most caudal part of the CLM, smooth muscles called the "corrugator cutis ani" traverse the distal anal sphincter into the perianal skin and the ischiorectal fossa. Those smooth muscles also pass through the IAS to intersect within the subepithelial space. Any disruption of these muscles will bring about anal cushion prolapse, vascular outflow obstruction, and hemorrhoidal bleeding and thrombosis - this is the well-known theory of symptomatic hemorrhoid disease [14]. The pathway of the corrugator cutis ani should be understood during a hemorrhoidectomy. Dissection should be done between the hemorrhoidal plexus and the IAS, not deep into IAS or even the CLM and the EAS. Therefore, lidocaine with epinephrine injection might be helpful in defining the anatomical dissection plane with adequate hemostasis.

\section{EXTERNAL ANAL SPHINCTER}

The EAS consists of a striated muscle that envelops the entire IAS and CLM. The EAS is approximately $2.7 \mathrm{~cm}$ high, but is anteriorly shorter in women, being approximately $1.5 \mathrm{~cm}$ [4]. The EAS is considered to have one or more subdivisions, although the precise composition is disputed. Milligan and Morgan [15] insisted the 

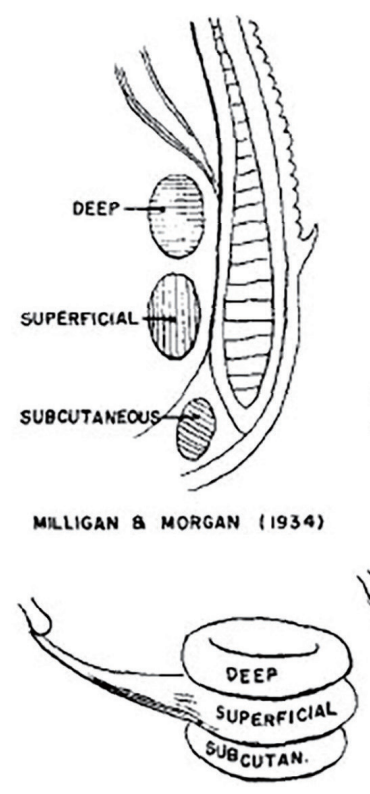

GABRIEL (1948)

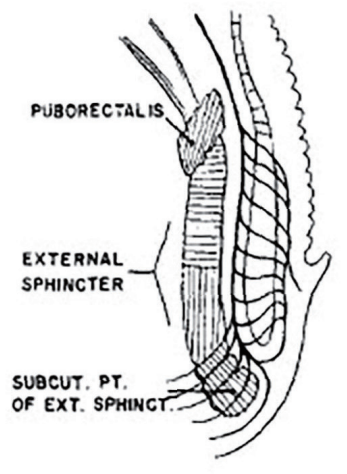

GOLIGHER (1967)

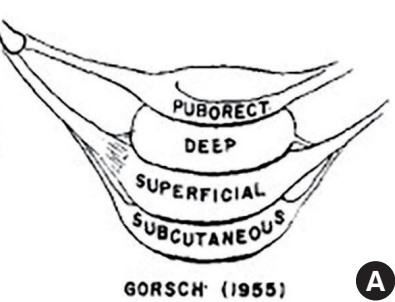

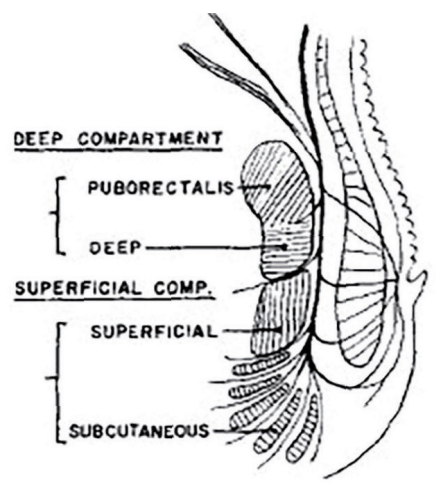

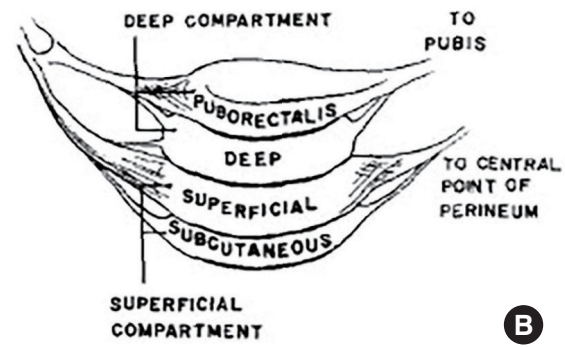

Fig. 2. Diagrams of the external anal sphincter comparing previous views (A) with Oh and Kark's findings (B). (A) Coronal views show two kinds of sphincteric arrangements - trilaminar and bilaminar patterns. The lateral views show the anteroposterior attachment of the external sphincter. (B) The deep compartment (puborectal muscle and deep sphincter) and the superficial compartment (superficial and subcutaneous sphincter) based on histological sections and on the pattern of anteroposterior attachment shown by gross dissection. Reproduced from Oh and Kark. Br J Surg 1972;59:717-23, with permission of John Wiley and Sons [17].

EAS has three distinct divisions, which are the Sphincter ani externus subcutaneous, superficialis, and profundus. However, Goligher et al. [16] failed to demonstrate these three parts of the EAS and described the EAS as one continuous sheet that is fused with the puborectalis. Oh and Kark [17] examined 48 cadaveric and surgical specimens and concluded that the arrangement and the distinction between the subdivisions are not the same at different planes. They also argued that describing the EAS as being composed of a deep compartment (deep sphincter and puborectalis) and a superficial compartment (subcutaneous and superficial sphincter) would be more appropriate (Fig. 2).

Shafik [18] suggested a triple loop system. The top loop (deep part of the EAS and puborectalis) wraps the upper part of the rectal neck and is attached to the symphysis pubis, which is innervated by the inferior hemorrhoidal nerve. The intermediate loop and the basal loop embrace the mid portion and the lower rectal neck, respectively. In addition, the perineal branch of the fourth sacral nerve and the inferior hemorrhoidal nerve innervated them, separately. Even though this concept and its functional interpretation are innovative, they are not generally accepted.

\section{PERINEAL BODY}

The perineal body $(\mathrm{PB})$ is a fibromuscular mass between the upper end of the anterior anal canal and the posterior wall of the urethral membrane in males and between the posterior wall of the vagina and the anal canal in females. This area is crucial to the urologist, as well as to the proctologist who performs various types of perineal surgery: an abdominoperineal resection (APR), pull-through procedures, and repair of a rectovaginal fistula or a urethrorectal fistula. It represents the intersection of the EAS, the bulbospongiosus muscle, the external urethral sphincter, and the levator ani muscle, as striated muscle. Smooth muscles such as the RUM, CLM, and IAS are anchored to the PB [19]. Kraima et al. [19] described the PB as being both centrally located between the anal and urogenital triangle and almost completely formed by muscle fibers derived from the CLM. They also described the PB as filling the anterior gap of the cranial part of the EAS.

That superficial, deep, transverse perineal muscles contribute to the composition of the $\mathrm{PB}$ is generally accepted. Nevertheless, the detailed description of the $\mathrm{PB}$ differs with the researcher. One study using thin-slice MRI reported that the PB consisted of the bulbospongiosus, the superficial transverse perineal muscle, the IAS, the EAS, the puboperinealis muscle, and the pubo-analis [20].

\section{RECTOURETHRALIS MUSCLE}

Henle defined the muscular bundle interposed between the anterior perineal flexure of the rectum and the membranous urethra 

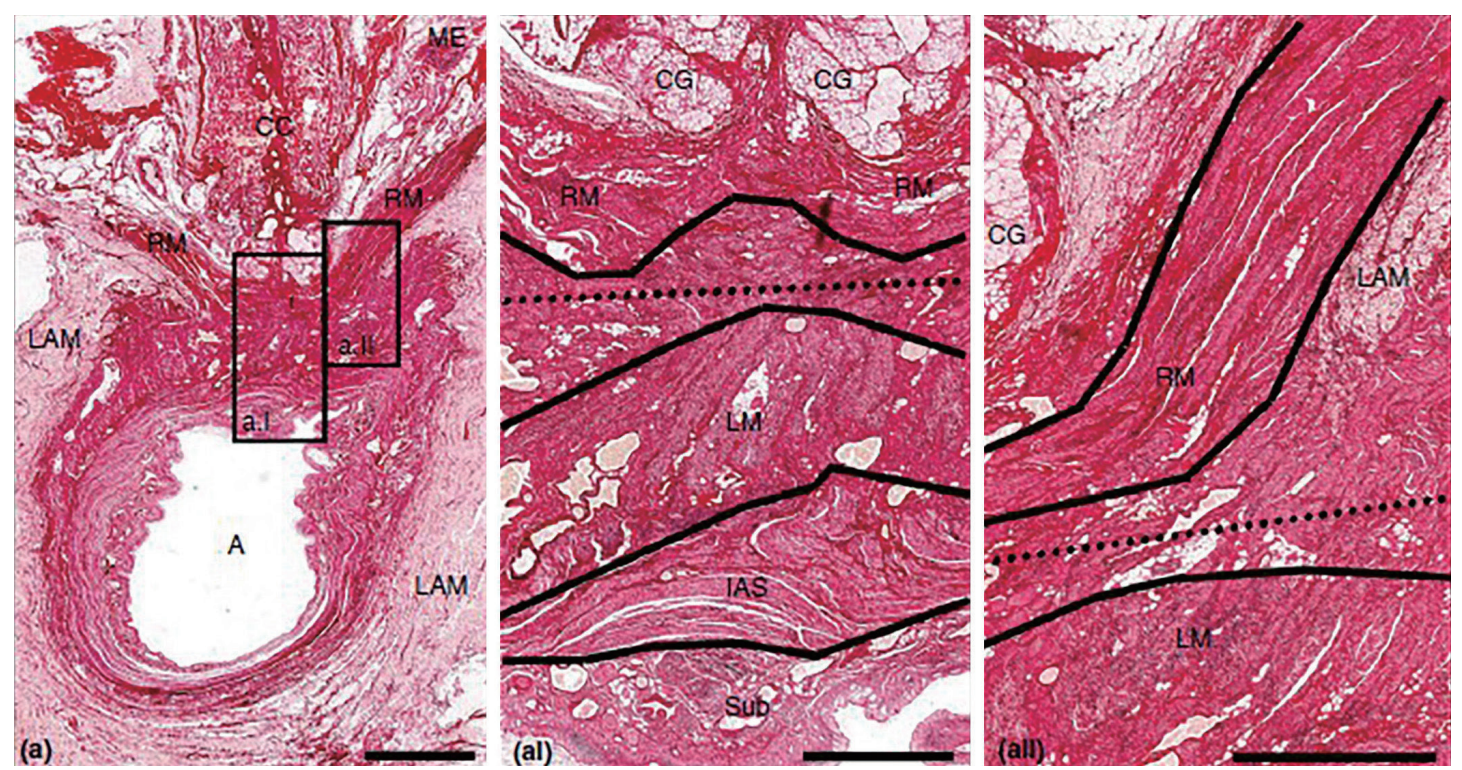

Fig. 3. Panel (a) shows a center of the male PB at the level of the anorectum A. Panel (aI) shows the PB bounded by the LM and the IAS. Anteriorly, the PB is bounded by the RM. Panel (aII) shows the relation of the RM to the LM and levator ani muscle. The dotted lines in panels (aI) and (aII) indicate the lines of dissection for a safe APR. Miller's elastin staining was used in all images. LM, longitudinal muscle; ME, Miller's elastin; PB, perineal body; RUM, rectourethralis muscle; APR, abdominoperineal resection. The scale bar in panel (a) = $6 \mathrm{~mm}$, and those in panels (aI) and (aII) $=2 \mathrm{~mm}$. Reproduced from Kraima et al. Colorectal Dis 2016;18:688-95, with permission of John Wiley and Sons [19].

as 'prerectal.' Roux was the first to use the term 'rectourethralis muscle' in reference to the prerectal muscle. It originates from the CLM, extends into the PB, and adheres anterolaterally to the levator ani muscle and posteriorly to the CLM [21]. Thus, it occupies the anterior portion of the rectum, which is not covered by the levator ani, and is located directly above the EAS [22].

According to Porzionato et al. [21], the RUM does not contact the posterior wall of the urethra. According to them, the mean distance between the RUM and the membranous urethra was 5.3 $\pm 1.25 \mathrm{~mm}$ in adults and $1.0 \pm 0.41 \mathrm{~mm}$ in infants. In infant specimens, the RUM was clearly separated from the levator ani while in 8 of 10 adults (80\%), the striated fibers of the levator ani and the smooth muscle fibers of the RUM intermingled. Those authors suggested that these structural associations might be related to functional cooperation between the 2 muscles.

As the RUM is located superior to the $\mathrm{PB}$, an understanding of this structure is essential to the surgeon performing a radical prostatectomy and an APR for prostate cancer and distal rectal cancer, respectively. Uchimoto et al. [22] showed that the cavernous nerve passed through the RUM and that the rectal vein drained into the venous plexus through the RUM. They recommended limited use of electrocauterization for hemostasis because those nerves and blood vessels might be damaged during an APR or an ISR. Furthermore, the abdominal surgical plane behind the DVF helps surgeons to guide their fingers along the external rectal muscularis propria. Palpating the external rectal muscularis propria should be helpful for adjusting the plane of the perineal approach for identifying the RUM. Moreover, Kraima et al. [19] emphasized that dissection should be started just anterior to the EAS and continue cranially, following the line between the RUM and the longitudinal muscle of the rectum. This not only preserves the autonomic nerves but also is oncologically safe when performing an APR (Fig. 3).

\section{PELVIC FLOOR}

The pelvic floor is a complex structure of muscle, ligaments, and fascia with various roles. Although often thought to be a single muscular layer, the pelvic floor is actually more complex. From superior to inferior, it is composed of the endopelvic fascia, the muscular pelvic diaphragm, and the perineal membrane [5]. As for the pelvic diaphragm, it consists of the levator ani muscle (puborectalis, pubococcygeus, and iliococcygeus) and the coccygeus. Some had argued that the puborectalis was part of the deep portion of the EAS. However, imaging and embryological study showed that the levator ani muscle has a distinct muscle fascicle direction and shares the same primordium. The levator ani muscle is innervated by pudendal nerve branches and the S3 and 4 nerves. The pubococcygeus and the puborectalis muscles are primarily innervated by pudendal nerve branches such as the inferior rectal nerve while the iliococcygeus is primarily innervated by the sacral S3 and S4 nerves (Fig. 4) [5].

The levator ani muscle is thick anteriorly and mostly thin aponeurotic posteriorly. This funnel-shaped pelvic diaphragm sus- 

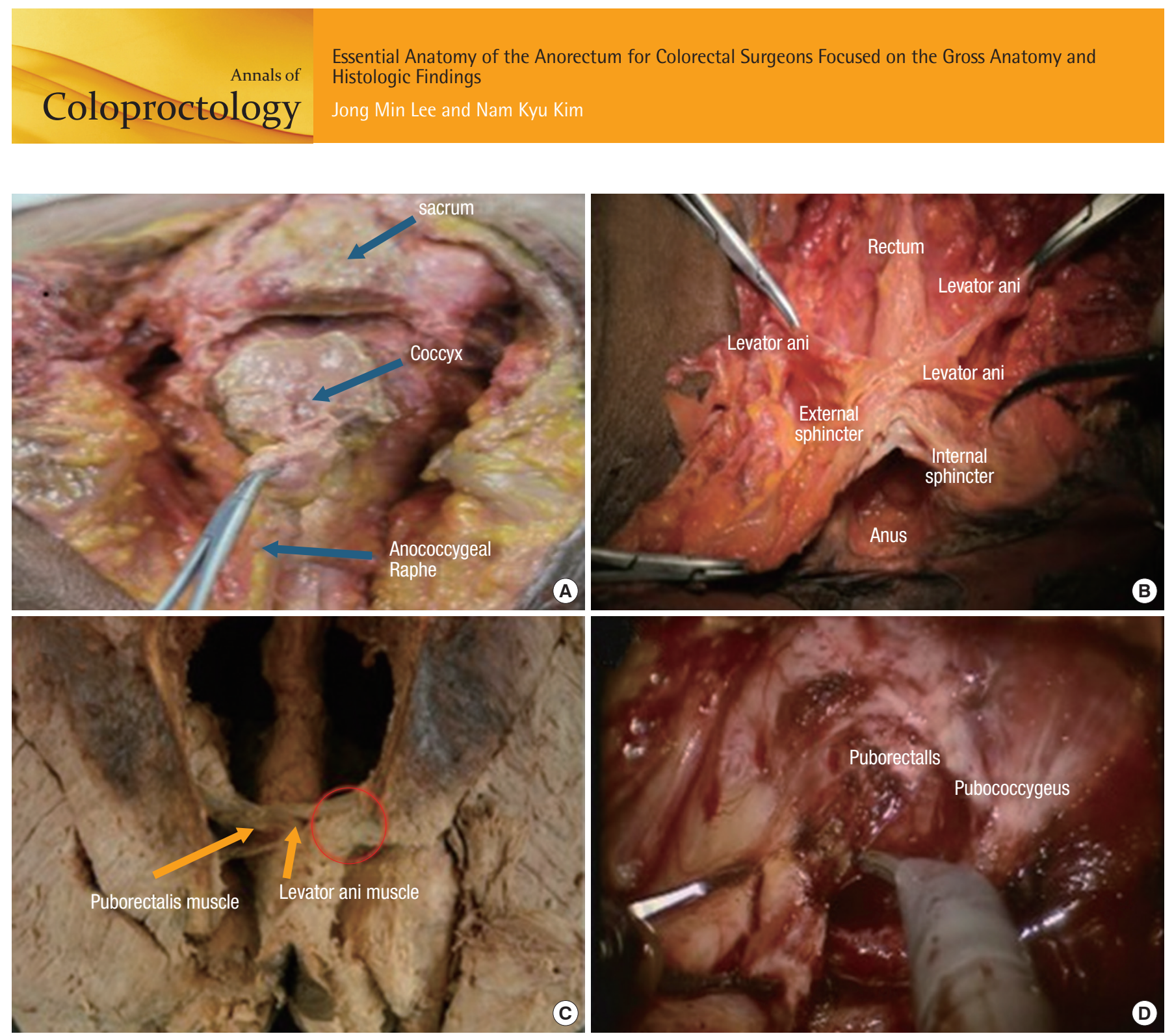

Fig. 4. Anatomy of the pelvic floor muscles. (A) Posterior aspect of the pelvis after division of the sacrum. (B) After removal of the sacrum, the funnel-shaped pelvic floor with the sphincter complex. (C) Fixed cadaveric specimen showing the pelvic floor and the puborectalis muscle. (D) Exposure of the pelvic floor muscles (puborectalis and pubococcygeus) after deep pelvic dissection.

pends the rectum that is located just above the pelvic floor. Based on personal experience with rectal MRI, various shapes of pelvic floor, which may differ according to sex and BMI, may be observed. Its superior attachment is on the posterior surface of the pubic bone anteriorly and on the internal obturator muscle and the ischial spine laterally. Posteriorly, the levator ani muscle borders the coccygeus and the anococcygeal raphe (Fig. 5).

The pubovisceral muscle, which comprises the puborectalis and the pubococcygeus, is located medially and superiorly. The pubovisceral muscle was renamed because of its role in the suspension of the distal gut [23]. The pubovisceral muscle's origin is transparent and thin as it attaches tangentially to the pubic periosteum and runs laterally from the tendinous arch and ischial spine [24]. The medial part is continuous inferiorly with the $\mathrm{PB}$ and the CLM and is attached to the rectococcygeal fascia [23].

The clinical implication of the pelvic floor's anatomy in rectal cancer is the acuteness of the angle between the lateral wall of the levator ani muscle and the rectal wall, which may be related to the width of the pelvic floor, and that presents a working-space problem when dissecting the distal part during a TME. When an advanced low-lying rectal tumor is encountered, an APR or an extralevator APR should be considered so that a sufficient circumferential resection margin is obtained. In other words, if a need exists to excise the levator ani muscle at its origin, unless the anatomy of the pelvic floor is fully understood, an adequate extralevator APR cannot be performed. Actually, when the patient is in the jack-knife position, the levator ani muscle is more expanded and more visible. For an extralevator APR, some surgeons insist that the jack-knife position is necessary. Actually, circumferential margin positivity and tumor cell spillage from a perforated site are the main reasons for the poor oncologic outcomes following a standard APR for low rectal cancer. Therefore, an extralevator or a to- 

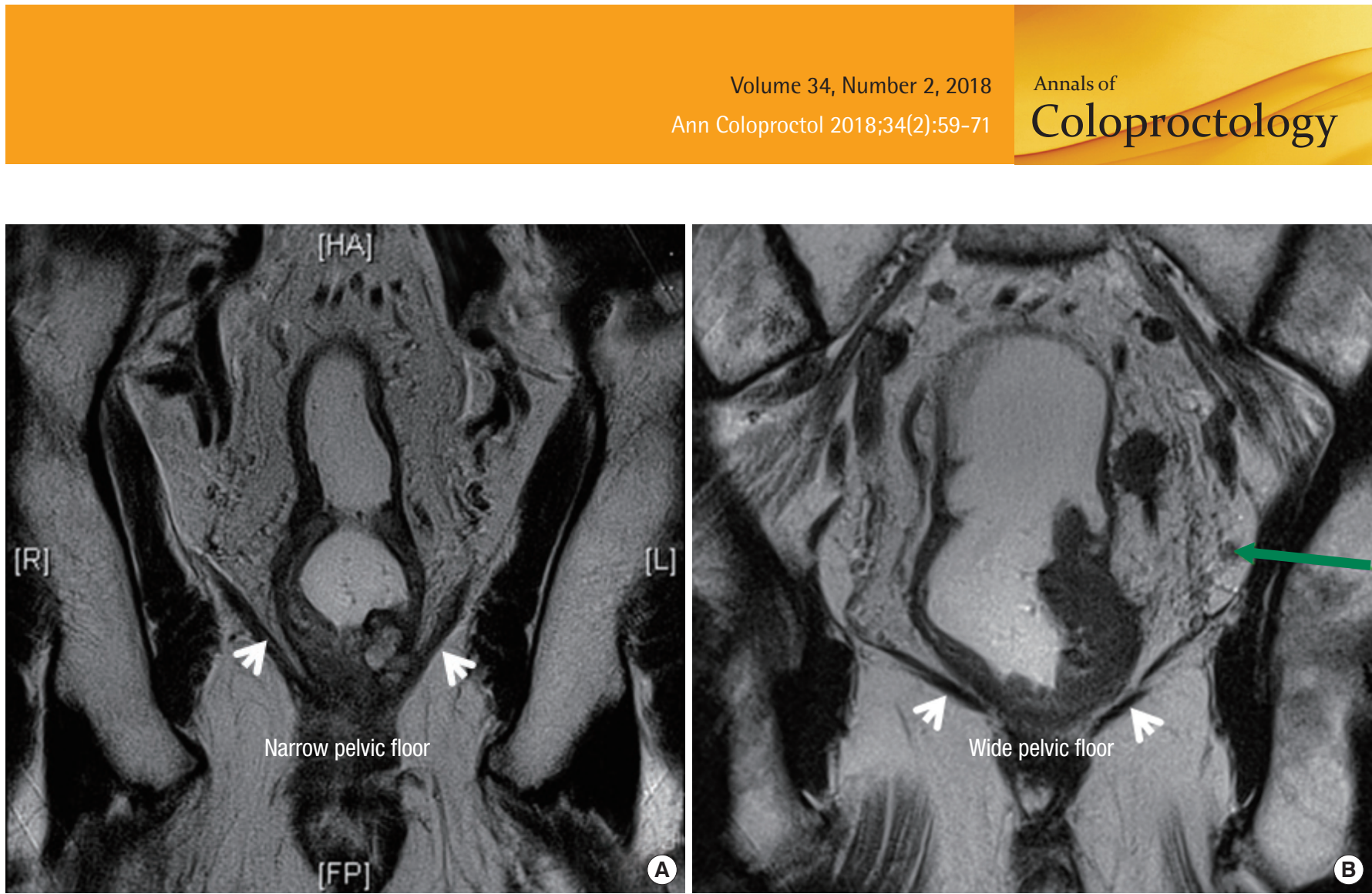

Fig. 5. Pelvic floor images on the coronal axial view of a magnetic resonance image. (A) A steep coning down of the pelvic floor with a narrow angle. (B) A gradual coning down of the pelvic floor with a wide angle.

tal levator excision approach has been developed to avoid coning down of the specimen. For an understanding of the correct surgical anatomical planes for these procedures, the pelvic floor's anatomy is essential. An en bloc resection of the pelvic floor with the mesorectal fascia (MRF) is important. That means the mesorectum should not be dissected off the levator ani muscles. Getting a cylindrically shaped specimen is important, which is the way to obtain a negative circumferential margin.

\section{RECTUM, MESORECTUM, AND PELVIC FASCIA}

Where the rectum starts is controversial because of the difference between the surgical and the anatomical anal canals. For surgeons, the rectum will be from the anorectal ring whereas for the anatomists, it will be from the dentate line. The rectum extends 12 to $15 \mathrm{~cm}$ proximally. The rectum can be distinguished from the sigmoid colon where the outer longitudinal muscle begins to form taenia coli. The rectum has 2 or 3 curves in the lumen, and those submucosal folds are called 'Valves of Houston. From the anal verge, the rectum can be defined to have 3 parts: the lower rectum, $0-6 \mathrm{~cm}$; the middle rectum, 7-11 cm; and the upper rectum, $12-15 \mathrm{~cm}$ [25]. The lower third of the anterior portion is extraperitoneal while the posterior part is totally extraperitoneal. The mesorectum is a fatty tissue encircling the rectum and is a very important structure in a total mesorectal excision for rectal cancer. It contains the possible metastatic lymph nodes and blood vessels and is enveloped with clear distinct collagenous fiber, the so-called 'mesorectal fascia', in other words, the fascia propria. The mesorectum is thick on the posterior and lateral side of the rectum, but on the anterior side, it is thinner than it is on the other sides. The mesorectum is well known to be almost absent at approximately $2 \mathrm{~cm}$ above the puborectalis muscle [26]. The MRF corresponds to the visceral fascia and seems to be thicker on the posterior side of the rectum. The parietal fascia refers to the membrane that adheres to the periosteum over the sacrum (presacral fascia) (Fig. 6) [27]. Knowing the spatial relationship between the MRF and surrounding structures, such as the inferior hypogastric nerve and the pelvic plexus, during a TME cannot be emphasized enough. The 'holy plane' of a TME is an avascular area bordered by the MRF and the parietal fascia.

Kraima et al. [28] demonstrated that no extra constant fascial layer existed between the MRF and the parietal fascia. The MRF is a multilayered envelop that shows gaps at some points. The interruption of the MRF is more pronounced at the lower anterolateral mesorectum. The autonomic nerves are located directly lateral to the MRF, but within the parietal fascia (Fig. 7). Thus, if the mesorectum is not to be breached for oncologic reasons and at the same time, the nerves are not to be damaged to save the function, a TME should be carried out using a sharp dissection on the MRF. In other words, the closer the TME is performed to the MRF, the greater is the likelihood of autonomic nerve preservation.

At the S4 level, relatively dense connective tissue links the presa- 


\section{Annals of Histologic Findings \\ Coloproctology Jorg Min ke and Nam Ku kim}

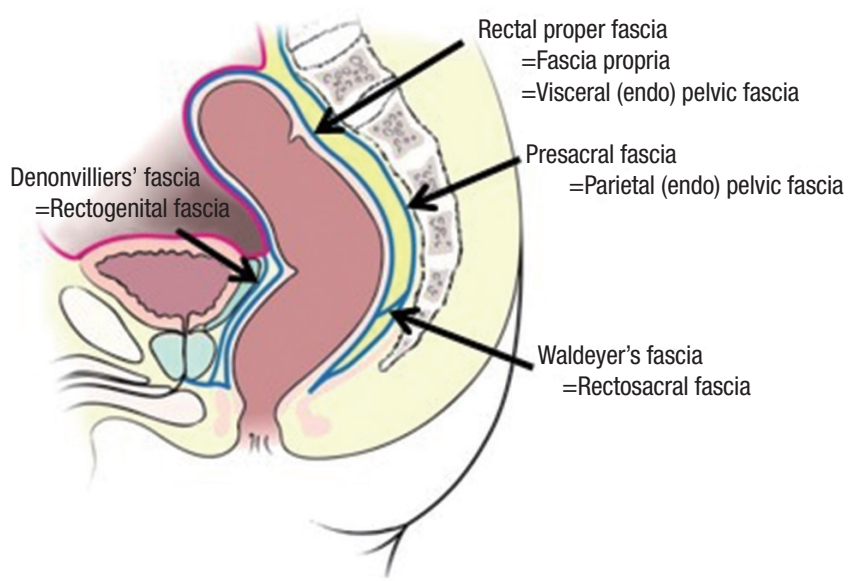

Fig. 6. Locations of the rectum in the pelvic cavity and of the normal fascia structures around the rectum. The rectal proper fascia, the fascia covering the mesorectum, is also called visceral endopelvic fascia; the presacral fascia, the fascia covering the sacrum, is also called the parietal endopelvic fascia; Denonvilliers' fascia, a dense membrane between the rectum and the seminal vesicles, is also called the rectogenital fascia; Walderyer's fascia is a dense connective tissue layer between the posterior part of the rectal proper fascia and the presacral fascia at the levels of S3 and S4.

cral fascia and the MRF. This is called the rectosacral fascia (Waldeyer's fascia). Crapp and Cuthbertson [29] pointed out the clinical significance of this because it causes bleeding in the presacral venous plexus unless it is recognized and divided. When it is thick, a blunt dissection with the hand can cause avulsion injury to the presacral vein. A sharp division of the rectosacral fascia allows the surgeon to arrive at the level of the coccyx. If this fascia is sharply divided, dissection can enter the deep pelvic cavity; then, the gate to the pelvic floor will surely be met and the rectal wall (in this area, no mesorectum exists) will easily be separated from the pelvic floor. A better approach is to start the anterior pelvic dissection after doing the posterior dissection as much as possible. From a surgeon's practical point of view, such anatomical knowledge and concepts are very important.

The DVF is present between the seminal vesicle and the rectum and forms a shiny, white surface. It is present as a rectovaginal septum in females. Debate exists about whether to enter anterior or posterior to the DVF. Lindsey and Mortensen [30] asserted that the mesorectal plane, immediately outside the MRF, is the anatomical plane that can minimize sexual dysfunction. They insisted the dissection should be performed between the mesorectal fascia and the DVF. However, Heald et al. [31] stated that a dissection behind the DVF was difficult and oncologically suboptimal while a dissection at the anterior plane to the DVF, called the extramesorectal plane, was natural and bloodless. He stated that a gentle backward pressure on the specimen helped the surgeon to enter the areolar tissue anterior to the DVF. According to Kraima

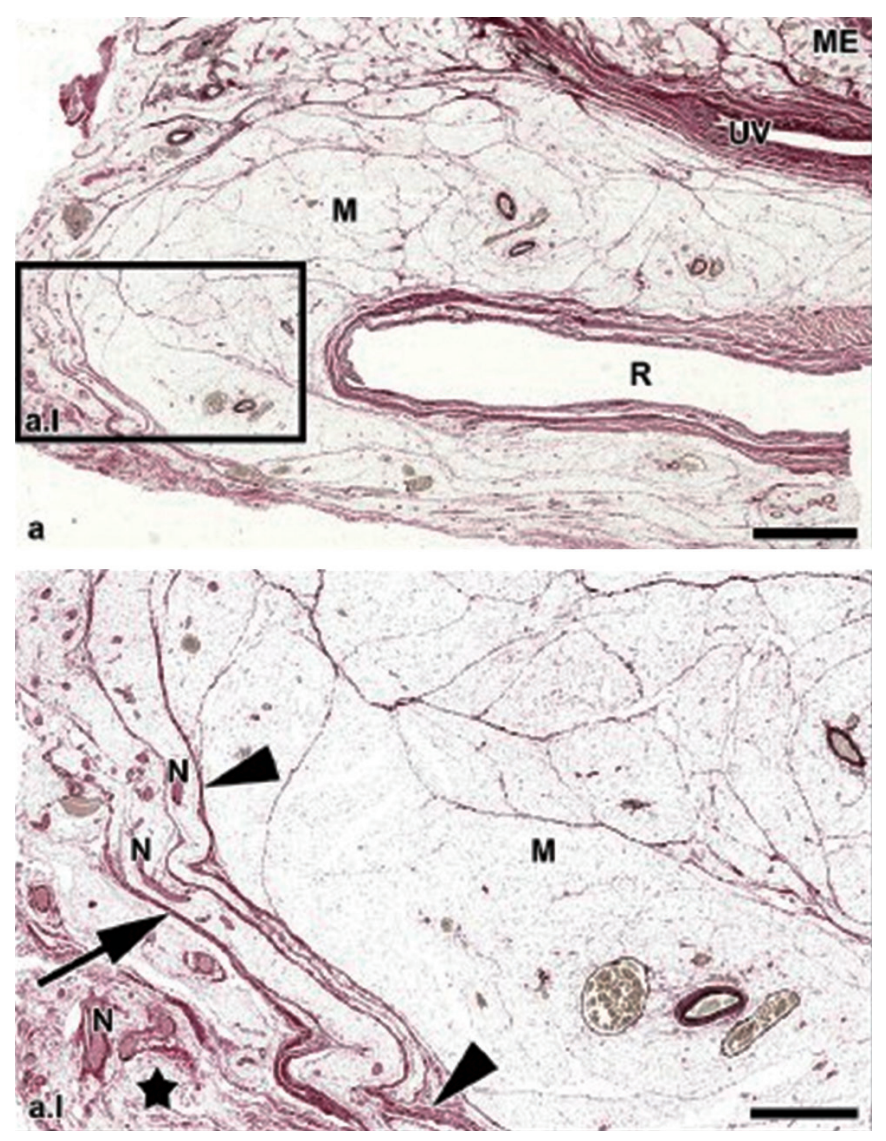

Fig. 7. Images showing the structures of the fasciae posterolateral to the mid rectum $\mathrm{R}$. The parietal fascia (arrow) covers the presacral space (star) which contains the pelvic splanchnic nerves N. The mesorectal fascia (arrowheads) consists of multiple laminae. Note that the autonomic nerves $\mathrm{N}$ run between the mesorectal fascia and the parietal fascia. UV, upper vagina; $\mathrm{M}$, mesorectum; ME, Miller's elastin. The scale bar in panel a is $6 \mathrm{~mm}$, and that in panel a.I is $2 \mathrm{~mm}$. Reproduced from Kraima et al. Eur J Surg Oncol 2015;41:1621-9, with permission of Elsevier [28].

et al. [32], the DVF begins at the top of the seminal vesicle and terminates in the PB. It consists of multiple fascial condensations of collagen and smooth muscle. Using whole mount microscopic sections of cadavers, they observed that the DVF was adhered to the posterior surface of the seminal vesicle and was indistinguishable from the MRF. At a more inferior level, some intervening space filled with adipose tissue existed between the prostatic fascia and the MRF at the posterolateral side of the prostate. However, the DVF was continuous with the MRF and the posterior prostatic fascia in the middle of the prostate, indicating that they were fused. Kinugasa et al. [33] described them as communicating branches of the bilateral pelvic plexus in front of the DVF, which compensated for each other. Lateral to the seminal vesicle, the DVF divided into multiple lamiae, and one layer of the DVF extended dorsolaterally, separating the MRF from the pelvic 

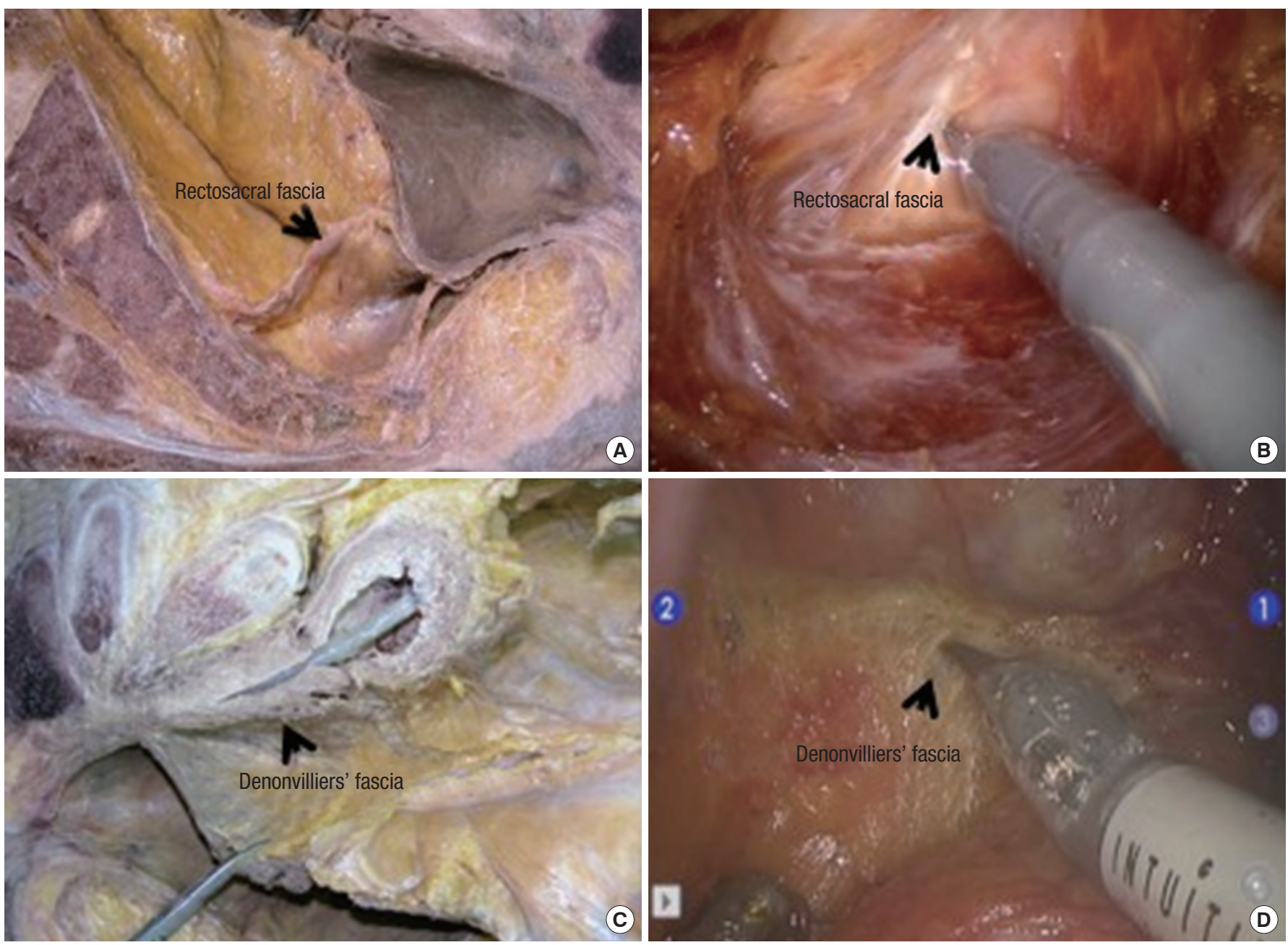

Fig. 8. Anatomy of the fascia. (A) Rectosacral fascia connecting the presacral fascia at the level of S3 and the rectal proper fascia in a cadaveric hemipelvis dissection. (B) Rectosacral fascia encountered during robotic surgery. (C) Denonvilliers' fascia in a cadaveric hemipelvic dissection. (D) Denonvilliers' fascia encountered during robotic surgery.

plexus. In a histologic evaluation using fresh cadavers, when the dissection plane was in front of the DVF, it extended laterally to the pelvic plexus. However, when the surgical plane started behind the DVF, the DVF and the pelvic plexus were clearly separated from the mesorectum.

Based on our experience, performing the anterior part of a TME following the mesorectal plane, as Lindsey proposed, is possible [30]. The extramesorectal plane is associated with a high risk of intraoperative hemorrhage and nerve injury. Unless the cancer is located at the anterior wall of the rectum and obtaining a sufficient margin is not at risk, we prefer to follow the mesorectal plane. However, in situations where a dissection is needed along the extramesorectal plane, we carefully use a U-shaped cut at the lateral border of the DVF to minimize damage to the neurovascular bundles (NVBs) (Fig. 8).

\section{PELVIC PLEXUS AND NEUROVASCULAR BUNDLE}

As the nerves from the preaortic plexus go down to the pelvis, they form the superior hypogastric nerve plexus at the level of the sacral promontory. The hypogastric nerve begins where the superior hypogastric nerve divides right and left. Each hypogastric nerve descends inferiorly and laterally and joins the pelvic splanchnic nerve (S2-4) to form the pelvic plexus. The pelvic plexus is known to be located on the lateral side of the pelvic wall and away from the parietal fascia. One study that examined 12 autopsied cadavers showed that the pelvic plexus was located, on average, from $3.3 \mathrm{~cm}$ above to $2.3 \mathrm{~cm}$ below the peritoneal reflexion [34]. Numerous small NVBs running from the pelvic plexus to the genitalia cross the seminal vesicles in the 10 oclock and 2 o'clock directions. Y-shaped pelvic autonomic nerve structures are 

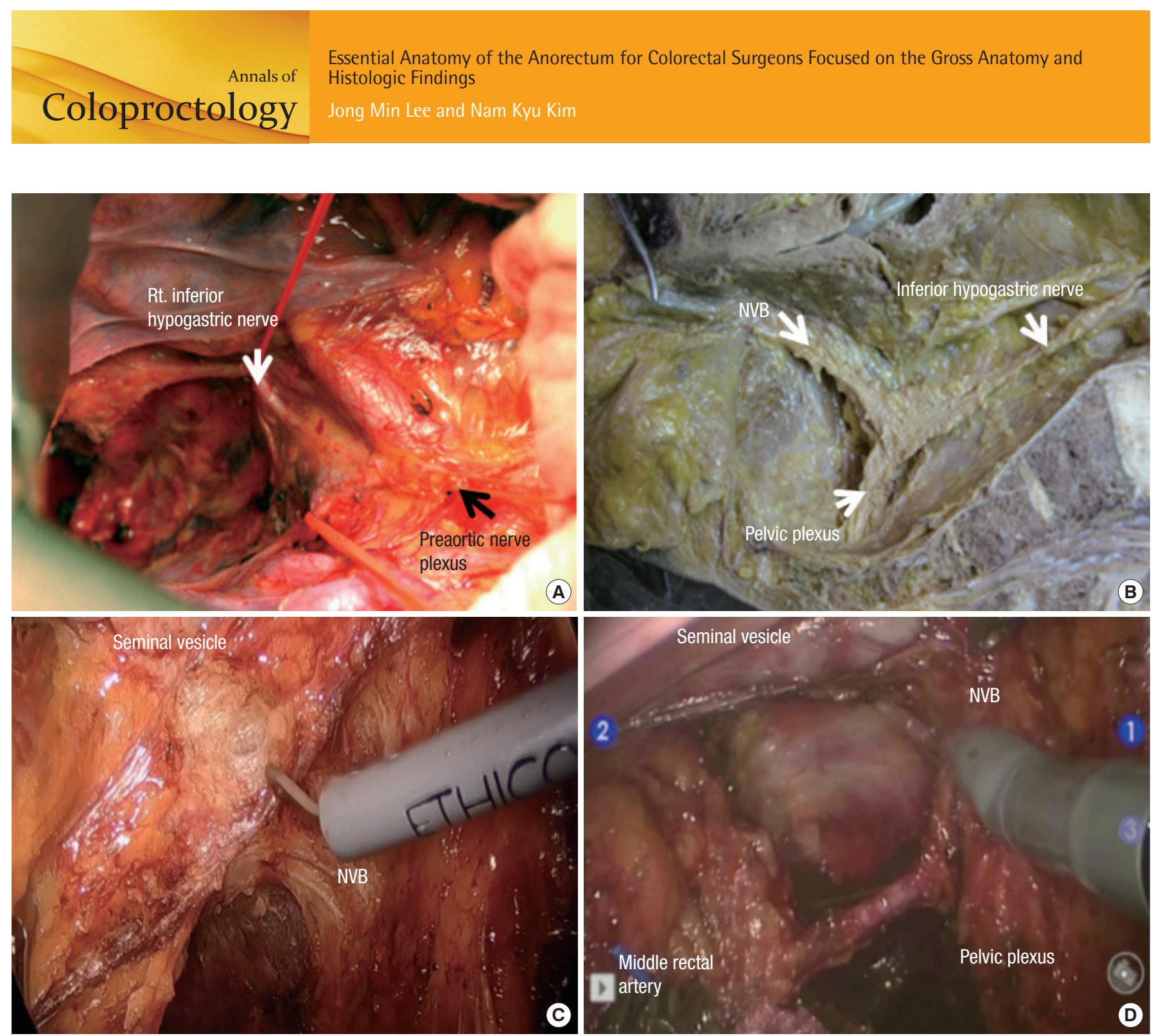

Fig. 9. Pelvic autonomic nerve structures. (A) Both inferior hypogastric nerves at the sides of the pelvic wall, going down to the deep pelvis, merge with the sacral parasympathetic nerves from S2-4 and finally form the pelvic plexus with a mesh-like structure. (B) Y-shaped pelvic autonomic nerve structures seen in the hemipelvis of a cadaveric section, showing that the inferior hypogastric nerve descends along each side of the pelvic wall and merges with the sacral parasympathetic nerves to become the pelvic plexus. This is densely attached to the lateral part of the mesorectal fascia, and the NVBs extend to the genitalia. (C) Laparoscopic view of the pelvic plexus and NVBs. (D) A robotic view of the middle rectal artery arising near the pelvic plexus and piercing the pelvic plexus. The Denonvilliers' fascia continues with the right side of the NVBs, which arise from the pelvic plexus. These structures can be seen after full mobilization of the rectum from the underlying pelvic floor muscles. NVBs, neurovascular bundles.

clearly seen at the hemipelvis during cadaveric dissection (Fig. 9) [35].

Performing the TME so as to avoid injury to the pelvic plexus after initiating an anterolateral pelvic dissection is important. The MRF is directly adhered to the mesh like the pelvic plexus; this adhered portion used to be regarded as a ligament, the so-called lateral ligament. This ligamentous structure between the mesorectum and the inferior hypogastric nerve and the pelvic plexus varies in thickness. This lateral adhesion may contain a middle rectal artery. The middle rectal artery was found in only $22.2 \%$ of the cases, and in those cases, the diameter of the artery was 1.6 $\mathrm{mm}$. Its origin was located $32.8 \mathrm{~mm}$ from the origin of the superior vesical artery, $19.9 \mathrm{~mm}$ from the upper border of the coccygeus muscle. The pelvic splanchnic nerves from the third and the fourth sacral nerves, which arise more posteromedially than the middle rectal artery, can be considered as components of the lateral ligament [36].

Regarding injury to the pelvic plexus, the surrounding areas should be handled carefully during mobilization of the rectum from the pelvic cavity. However, if the tumor is located near, or even invades, the pelvic wall, an en bloc resection with the pelvic plexus should be performed. Yamakoshi et al. [34] reported inter- 


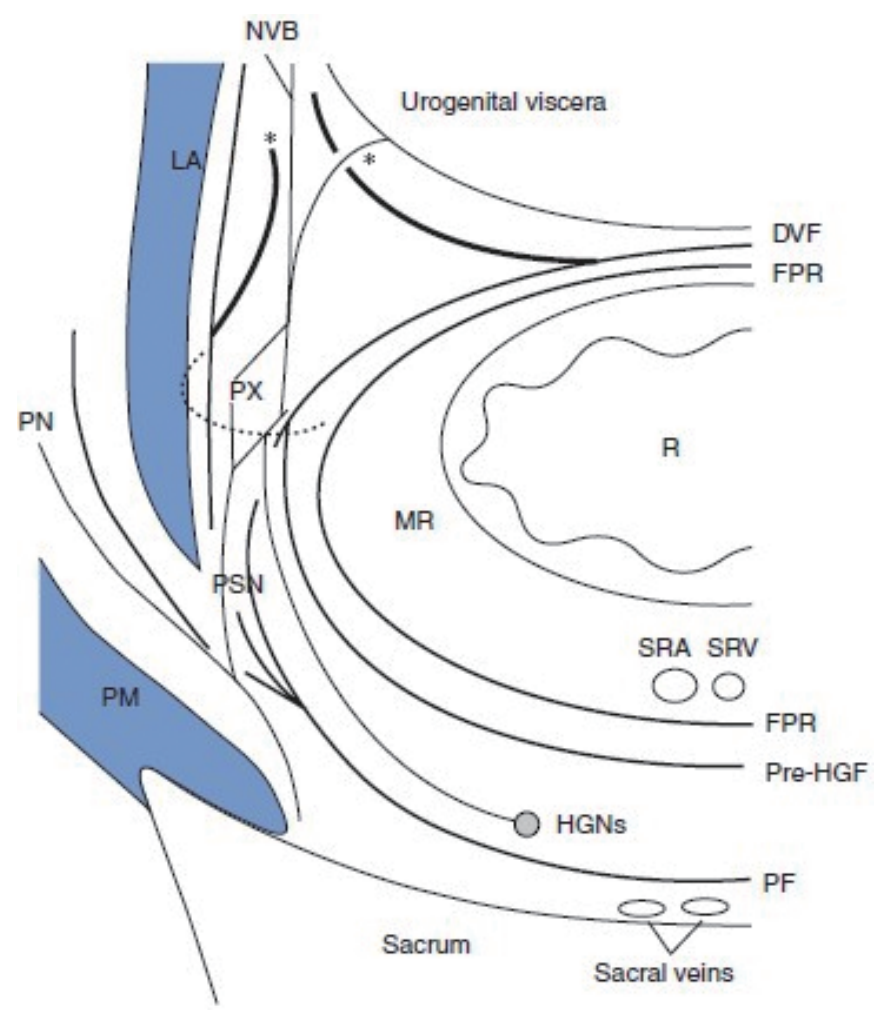

Fig. 10. Schematic relations between the pelvic plexus, neurovascular bundles and fascia. FPR, fascia propria of the rectum; HGN, hypogastric nerve; $\mathrm{MR}$, mesorectum; $\mathrm{PM}$, piriformis muscle; $\mathrm{PN}$, pudendal nerve; pre-HGF, pre-HGN fascia; PSN, pelvic splanchnic nerve; PX, pelvic plexus; R, rectum; SRA, superior rectal artery; SRV, superior rectal vein. Reproduced from Kinugasa et al. Br J Surg 2007;94:20-6, with permission of John Wiley and Sons [40].

esting data with the mean distances between the muscularis propria and the pelvic plexus in both autopsied and surgical specimens being $8.3 \mathrm{~mm}$ and $14.7 \mathrm{~mm}$, respectively. The distance between the deepest point of the cancer and the pelvic plexus in pT3 patients was $5.4 \mathrm{~mm}$, on average. This discovery led us to consider whether to resect the pelvic plexus concomitantly when middle or low rectal cancer has invaded deeply beyond the muscularis propria.

The NVBs run between the rectum and the posterolateral surface of the prostate. As described by Walsh and Schlegel [37, 38], they course in front of the rectogenital fascia or in the space occupied by the seminal vesicles and the prostate gland. The inferior branches of the pelvic plexus join with several vessels to form NVBs [39]. Based on Walsh's report on a nerve-sparing radical prostatectomy, the seminal vesicles can be used as an intraoperative landmark to identify the pelvic plexus, which is punctured by branches of the inferior vesical artery and veins and is embedded in the thick fascia (Fig. 10).

During the pelvic dissection, the importance of avoiding dam- age to the pelvic plexus and the NVBs should be emphasized. If the lateral aspect of the rectum is to be successfully resected from the pelvic plexus, incising the rectosacral fascia for a meticulous dissection down to the coccyx and separating the MRF from the pelvic plexus are important. Proper traction of the rectum is important to prevent avulsion injury to the pelvic plexus. During the dissection of this area, the middle rectal artery is occasionally seen, and it should be ligated with surgical clips or electrocauterized. Mass ligation and too much traction can damage the third sacral nerve, which is important for erectile function. Cutting the rectosacral fascia and opening the retrorectal space usually reveals the largest nervi erigentes of the S3 component. A sharp dissection around this area is necessary, but is difficult to perform in the deep and narrow male pelvis. However, the use of gentle traction with the magnified view of a robotic instrument will make preserving these structures easier compared to open surgery for the treatment of rectal cancer.

\section{ANORECTAL SPACES}

Anorectal spaces usually refer to the spaces that border various myofascial structures such as the levator ani, obturator internus, IAS, and EAS. Anorectal spaces are important to the colorectal sutgeons for the treatment of anorectal abscesses rather than they contained other meaningful structures.

The largest anorectal space is the ischiorectal space. The ischiorectal space forms an ischiorectal fossa with the perianal space that is below the ischiorectal space. The fascia starts from the CLM and reaches out to the ischial tuberosity across the subcutaneous EAS and the rest of EAS, which is the boundary between the perianal space and the ischiorectal space [41]. This fascia is the so-called 'transverse septum of ischiorectal fossa.' The medial border of the ischiorectal space is formed by the puborectalis, pubococcygeus, and deep EAS. The lateral border consists of the obturator fascia and ischium. Anteriorly, this area is bordered by the urogenital diaphragm and the transverse perineal muscle [41]. Most of the ischiorectal space is filled with large lobules of fat. The pudendal nerve and vessels locate superolaterally in this space.

The perianal space means the lower part of the anal canal surrounded by the anoderm. It is in contact laterally with the fatty tissue of the buttock and medially with the intersphincteric space. This space is a common site for perianal abscesses or hematomas. The intersphincteric space lies between the IAS and the EAS. It is filled with areolar tissue and fat. This space is continuous cephalad to the supralevator space that is situated between the peritoneum and the levator ani muscle. As described earlier, the anal gland secretes into the anal canal through the anal crypt along the level of the dentate line. Because some anal glands are in the intersphincteric space, this space plays a major role in the development of perianal fistulae and accounts for about half of the total incidence.

The superficial postanal spaces are interposed between the skin 
and the anococcygeal ligament. The deep postanal spaces occupy the region between the levator ani and the anococcygeal ligament. Superficial and deep postanal spaces communicate with the ischiorectal fossa and become locations where the horseshoe abscesses can occur. The inflammatory process may communicate between the ischiorectal fossa via the deep postanal spaces (space of Courtney). The retrorectal space is located between the mesorectal fascia and the presacral fascia. This space is continuous with the retroperitoneum upwards.

\section{CONCLUSION}

We reviewed the basic anatomy of and recent research results on the anorectum and looked at the strategy of surgery from the perspective of the surgeon. Until recently, anatomists have been actively studying the anatomy of this area and have been able to confirm the detailed analysis and new interpretation of the ambiguous structure. In particular, the study of the complex structure of the RUM and the PB seems to have given surgeons a new perception of the dissection plane. When the APR is implemented, preservation of the RUM without damage to the carvernous nerve or veins pass through the RUM is important. In addition, when the TME is performed, autonomic nerves pass immediately outside the MRF, so a more elaborate dissection is necessary. Also, performing dissection on the mesorectal plane in terms of autonomic nerve preservation is reasonable because the connecting branches of the pelvic plexus pass anterior to the DVF. We recommend that incise along the extramesorectal plane when CRM positivity is expected. Having adequate knowledge of anorectal anatomy is the most important factor for improving the oncologic and the functional outcomes in patients. Oncologic surgical principles for cure and function preservation based on anatomical knowledge and an updated minimal invasive surgical technique are essential for surgeons, so this trinity of surgical science must be mastered. However, because anorectal anatomy is not yet fully understood, we hope that additional studies on its anatomy will enable anorectal surgery to be performed based on complete anatomical knowledge.

\section{CONFLICT OF INTEREST}

No potential conflict of interest relevant to this article was reported.

\section{REFERENCES}

1. Nivatvongs S, Stern HS, Fryd DS. The length of the anal canal. Dis Colon Rectum 1981;24:600-1.

2. Tsukada Y, Ito M, Watanabe K, Yamaguchi K, Kojima M, Hayashi $\mathrm{R}$, et al. Topographic anatomy of the anal sphincter complex and levator ani muscle as it relates to intersphincteric resection for very low rectal disease. Dis Colon Rectum 2016;59:426-33.
3. Lilius HG. Fistula-in-ano, an investigation of human foetal anal ducts and intramuscular glands and a clinical study of $150 \mathrm{pa}-$ tients. Acta Chir Scand Suppl 1968;383:7-88.

4. Rociu E, Stoker J, Eijkemans MJ, Laméris JS. Normal anal sphincter anatomy and age- and sex-related variations at high-spatialresolution endoanal MR imaging. Radiology 2000;217:395-401.

5. Stoker J. Anorectal and pelvic floor anatomy. Best Pract Res Clin Gastroenterol 2009;23:463-75.

6. Haas PA, Fox TA Jr. The importance of the perianal connective tissue in the surgical anatomy and function of the anus. Dis Colon Rectum 1977;20:303-13.

7. Macchi V, Porzionato A, Stecco C, Vigato E, Parenti A, De Caro R. Histo-topographic study of the longitudinal anal muscle. Clin Anat 2008;21:447-52.

8. Shafik A. A new concept of the anatomy of the anal sphincter mechanism and the physiology of defecation. III. The longitudinal anal muscle: anatomy and role in anal sphincter mechanism. Invest Urol 1976;13:271-7.

9. Spanos CP. Intersphincteric resection for low rectal cancer: an overview. Int J Surg Oncol 2012;2012:241512.

10. Valadão M, Cesar D, Graziosi G, Leal RA. Operative technique: intersphincteric resection. J Coloproctol 2012;32:426-9.

11. Kinugasa Y, Moriya Y. Surgical anatomy in intersphincteric resection. In: Schiessel R., Metzger P. editors. Intersphincteric resection for low rectal tumors. Vienna: Springer-Verlag Wien; 2012. p. 57-63.

12. Noh GT, Han J, Cheong C, Han YD, Kim NK. Novel anal sphincter saving procedure with partial excision of levator-ani muscle in rectal cancer invading ipsilateral pelvic floor. Ann Surg Treat Res 2017;93:195-202.

13. Köhler A, Athanasiadis S, Ommer A, Psarakis E. Long-term results of low anterior resection with intersphincteric anastomosis in carcinoma of the lower one-third of the rectum: analysis of 31 patients. Dis Colon Rectum 2000;43:843-50.

14. Thomson WH. The nature of haemorrhoids. Br J Surg 1975;62: 542-52.

15. Milligan ET, Morgan CN. Surgical anatomy of the anal canal: with special reference to anorectal fistulae. Lancet 1934;2:1150-6.

16. Goligher JC, Leacock AG, Brossy JJ. The surgical anatomy of the anal canal. Br J Surg 1955;43:51-61.

17. Oh C, Kark AE. Anatomy of the external anal sphincter. Br J Surg 1972;59:717-23.

18. Shafik A. A new concept of the anatomy of the anal sphincter mechanism and the physiology of defecation. The external anal sphincter: a triple-loop system. Invest Urol 1975;12:412-9.

19. Kraima AC, West NP, Treanor D, Magee D, Roberts N, van de Velde CJ, et al. The anatomy of the perineal body in relation to abdominoperineal excision for low rectal cancer. Colorectal Dis 2016;18:688-95.

20. Larson KA, Yousuf A, Lewicky-Gaupp C, Fenner DE, DeLancey JO. Perineal body anatomy in living women: 3-dimensional analysis using thin-slice magnetic resonance imaging. Am J Obstet 
Gynecol 2010;203:494.e15-21.

21. Porzionato A, Macchi V, Gardi M, Parenti A, De Caro R. Histotopographic study of the rectourethralis muscle. Clin Anat 2005;18: 510-7.

22. Uchimoto K, Murakami G, Kinugasa Y, Arakawa T, Matsubara A, Nakajima Y. Rectourethralis muscle and pitfalls of anterior perineal dissection in abdominoperineal resection and intersphincteric resection for rectal cancer. Anat Sci Int 2007;82:8-15.

23. Wu Y, Dabhoiwala NF, Hagoort J, Shan JL, Tan LW, Fang BJ, et al. 3D Topography of the young adult anal sphincter complex reconstructed from undeformed serial anatomical sections. PLoS One 2015;10:e0132226.

24. Kim J, Betschart C, Ramanah R, Ashton-Miller JA, DeLancey JO. Anatomy of the pubovisceral muscle origin: macroscopic and microscopic findings within the injury zone. Neurourol Urodyn 2015;34:774-80.

25. Salerno G, Sinnatamby C, Branagan G, Daniels IR, Heald RJ, Moran BJ. Defining the rectum: surgically, radiologically and anatomically. Colorectal Dis 2006;8 Suppl 3:5-9.

26. Heald RJ, Husband EM, Ryall RD. The mesorectum in rectal cancer surgery--the clue to pelvic recurrence? Br J Surg 1982;69:6136.

27. Bissett IP, Hill GL. Extrafascial excision of the rectum for cancer: a technique for the avoidance of the complications of rectal mobilization. Semin Surg Oncol 2000;18:207-15.

28. Kraima AC, West NP, Treanor D, Magee DR, Bleys RL, Rutten HJ, et al. Understanding the surgical pitfalls in total mesorectal excision: investigating the histology of the perirectal fascia and the pelvic autonomic nerves. Eur J Surg Oncol 2015;41:1621-9.

29. Crapp AR, Cuthbertson AM. William Waldeyer and the rectosacral fascia. Surg Gynecol Obstet 1974;138:252-6.

30. Lindsey I, Mortensen NJ. Iatrogenic impotence and rectal dissection. Br J Surg 2002;89:1493-4.

31. Heald RJ, Moran BJ, Brown G, Daniels IR. Optimal total meso- rectal excision for rectal cancer is by dissection in front of Denonvilliers' fascia. Br J Surg 2004;91:121-3.

32. Kraima AC, West NP, Treanor D, Magee DR, Rutten HJ, Quirke P, et al. Whole mount microscopic sections reveal that Denonvilliers' fascia is one entity and adherent to the mesorectal fascia; implications for the anterior plane in total mesorectal excision? Eur J Surg Oncol 2015;41:738-45.

33. Kinugasa Y, Murakami G, Uchimoto K, Takenaka A, Yajima T, Sugihara K. Operating behind Denonvilliers' fascia for reliable preservation of urogenital autonomic nerves in total mesorectal excision: a histologic study using cadaveric specimens, including a surgical experiment using fresh cadaveric models. Dis Colon Rectum 2006;49:1024-32.

34. Yamakoshi H, Ike H, Oki S, Hara M, Shimada H. An assessment of the anatomical relationship between the pelvic plexus and the rectal wall to determine the indications for its preservation in surgery for rectal cancer. Surg Today 1997;27:1005-9.

35. Kim NK. Anatomic basis of sharp pelvic dissection for curative resection of rectal cancer. Yonsei Med J 2005;46:737-49.

36. Sato K, Sato T. The vascular and neuronal composition of the lateral ligament of the rectum and the rectosacral fascia. Surg Radiol Anat 1991;13:17-22.

37. Walsh PC, Donker PJ. Impotence following radical prostatectomy: insight into etiology and prevention. J Urol 1982;128:492-7.

38. Walsh PC, Schlegel PN. Radical pelvic surgery with preservation of sexual function. Ann Surg 1988;208:391-400.

39. Costello AJ, Brooks M, Cole OJ. Anatomical studies of the neurovascular bundle and cavernosal nerves. BJU Int 2004;94:1071-6.

40. Kinugasa Y, Murakami G, Suzuki D, Sugihara K. Histological identification of fascial structures posterolateral to the rectum. $\mathrm{Br}$ J Surg 2007;94:620-6.

41. Morgan CN. The surgical anatomy of the ischiorectal space. Proc R Soc Med 1949;42:189-200. 